\title{
LA ESTRATEGIA COMUNICACIONAL Y MEDIÁTICA DE RAFAEL CORREA
}

RAFAEL CORREA'S COMMUNICATION AND MEDIA STRATEGY

\author{
Punín Larrea, Ma. Isabel ${ }^{1}$ \\ Universidad Técnica Particular de Loja- Ecuador \\ mipunin@utpl.edu.ec \\ Rúas Araujo, José2. \\ Universidad de Vigo- España \\ xoseruas@gmail.com
}

Material original autorizado para su primera publicación en la revista académica REDMARKA. Revista Digital de Marketing Aplicado.

https://doi.org/10.17979/redma.2015.01.014.4882

Recibido: 13 Marzo 2015

Aceptado 10 Junio 2015

\footnotetext{
${ }^{1}$ Inicio su vida académica como profesor a tiempo parcial de la Universidad Técnica Particular de Loja ( Ecuador). En el 2007 cursó el Diplomado Superior Experto en Gestión y Calidad Universitaria. Universidad de Sevilla. Conocimiento que se permitieron acceder al cargo Coordinadora de la Titulación de Comunicación Social. Modalidad Abierta y Modalidad Presencial. Universidad Técnica Particular de Loja. ( UTPL - Ecuador, cargo que lo ocupa hasta mediados del 2014. En 2012 obtiene su titulo PhD en Comunicación y Periodismo. Universidad Santiago de Compostela. España. Ha sido conferencista invitada de la Universidad Luis de Sipán (Perú) y algunas otras universidades de Ecuador.

${ }^{2}$ profesor titular de "Técnicas de Comunicación Electoral e Institucional", en la Facultad de Ciencias Sociales y de la Comunicación de la Universidad de Vigo e investigador principal del grupo "Necom: neurocomunicación, publicidad y política" (necom.uvigo.es). Autor, entre otros, del libro Manual del Candidato Electoral. Madrid: Catarata (2011), así como de diversos artículos relacionados con la aplicación de las técnicas y herramientas de las neurociencias a la medición de la eficacia de los mensajes persuasivos.
}

REDMARKA UIMA-Universidad de A Coruña - CIECID

Año VIII, Número 14, (2015), v I pp. 75-101

http://www.redmarka.net/ ISSN 1852-2300 


\title{
Resumen:
}

La llegada de Rafael Correa a la presidencia de Ecuador en el año 2007 supuso una serie de cambios en la comunicación y el ejercicio periodístico. Se realiza un mapeo cronológico/descriptivo de las diversas acciones gobierno/medios.

Se estudia el impacto del discurso de Correa en los medios, las transformaciones del mapa mediático en Ecuador, y la estrategia comunicacional presidencial, tomando como referencia acontecimientos difundidos por la prensa nacional.

Las confrontaciones entre gobierno y medios privados genera un ambiente tenso, lleno de procesos judiciales y discusiones sin tregua, que han modificado el quehacer periodístico y con el la estructura de la empresa periodística en el país.

Palabras clave: Gobierno, sistemas de comunicación, medios de comunicación

\begin{abstract}
:
Rafael Correa's rise to power in 2007 implied a series of changes in communication and journalism. The impact of Correa's discourse in the mass media, along with transformations of the media map in Ecuador (a chronological/descriptive map of various government/media activities is included in this study), and an analysis of the communication strategy of the President are likewise provided, which use as a reference news events disseminated by the national press. The study highlights the various confrontations between the government and private media, which not only generated a tense political environment, but also led to legal proceedings and fiery debate. This subsequently led to changes in the journalism profession and in the restructuring of media companies in Ecuador.
\end{abstract}

Keywords: Government, communication systems, mass media

REDMARKA UIMA-Universidad de A Coruña - CIECID

Año VIII, Número 14, (2015), v I pp. 75-101

http://www.redmarka.net/ ISSN 1852-2300 


\section{Introducción:}

\section{Cambios políticos y mediáticos en Ecuador}

Analizar los cambios del mapa mediático en Ecuador resulta complicado y requiere de un análisis de las transformaciones de orden político y social. La estructura mediática en el país está conformada por medios comerciales y de carácter privado, los medios públicos de reciente creación, y los medios comunitarios "alrededor del $95 \%$ es de propiedad privada. El $5 \%$ restante se divide entre públicos y comunitarios" ( Abad, 2011:143) .

Rafael Correa Delgado llegó al poder, por primera vez, en el año 2007, usando una estrategia neo-populista y de corte socialista, cuyo lema más emblemático fue la "revolución ciudadana", en la cual Correa es el primer ciudadano de dicha revolución (Freidenberg, 2008).

El proceso liderado por Correa puso fin a un ambiente de inestabilidad política que vivió el país, y que acabó con la representación de partidos tradicionales como: Izquierda Democrática (ID), Concentración de Fuerzas Populares (CFP), Partido Social Cristiano (PS) y Movimiento Popular Democrático (MPD), entre otros. Correa usando la figura de outsider se benefició del discurso de la revuelta forajida y de la protesta ciudadana contra un sistema de partidos en crisis (Freidenberg, 2008, p. 185-233).

Un liderazgo que se mantuvo fortalecido a lo largo de sus dos periodos presidenciales, hasta la realización del pasado 23 de febrero del 2014, del proceso eleccionario de alcaldes, prefectos y demás autorizades seccionales, en

REDMARKA UIMA-Universidad de A Coruña - CIECID Año VIII, Número 14, (2015), v I pp. 75-101 http://www.redmarka.net/ ISSN 1852-2300 
el cual Correa pierde el respaldo de provincias fundamentales para su gestión como: Pichincha, Guayas, Cuenca y Loja3.

Desde el principio de su gestión, Correa realizó varios cambios que llamaron inmediatamente la atención de los medios de comunicación nacional e internacional. En primera plana de la prensa reseñó la eliminación de la intermediación laboral, que libró de responsabilidades a empresarios privados del país relacionadas con los derechos sociales de los trabajadores; la fijación de un salario mínimo para los trabajadores en $\$ 318$ dólares en el 2012, frente a los aproximadamente 180 y 250 dólares en los que se situaba hasta entonces; la modernización de escuelas, hospitales públicos, y la gestión vial a nivel nacional. Todas ellas han sido acciones estrategias de su gestión presidencial.

Estos y otros factores influyeron desde el principio para consolidar el perfil político del presidente Rafael Correa Delgado, perfil que fue ratificado por un respaldo mayoritario, como se puede ver en la siguiente tabla del Consejo Nacional Electoral (CNE). Dentro del ejercicio del poder presidencial se han realizado en el país dos elecciones generales, dos plebiscitos, una elección constituyente, procesos en los cuales el voto se ha inclinado mayoritariamente hacia la propuesta Correista; esto por supuesto antes del proceso electoral ya comentado anteriormente, en el cual se evidenció la poco aceptación popular que los ecuatorianos dieron a los candidatos del Movimiento Alianza País.

Por otro lado, el informe Latinobarmetro (2012) hace una evaluación de los líderes latinoamericanos, tomando en consideración la opinión ciudadana. Correa Delgado alcanza el 5.3\%, mientras que Barack Obama tiene el 6.3\%. Este mismo informe señala que el Presidente Ecuatoriano tuvo en el 2007 un nivel de

\footnotetext{
${ }^{3}$ El detalle de los resultados Elecciones 2014, se pueden visualizar la página oficial del Consejo Nacional Electoral (CNE) en la siguiente dirección:

http://resultados2013.cne.gob.ec/Results.html?RaceID=1\&UnitID=1\&IsPS=0\&LangID=0 ( 03/04/2014).

REDMARKA UIMA-Universidad de A Coruña - CIECID

Año VIII, Número 14, (2015), v I pp. 75-101

http://www.redmarka.net/ ISSN 1852-2300
} 
aceptación del 4.7\%, logrando una escalada hasta el 2011 de 5.3\% (Latibarómetro, 2012).

La escalada política de Correa Delgado se consolida en menos de una década. Uno de los factores de este ascenso es el uso de un discurso provocador que apela al convencimiento y a la esperanza; recurre a las emociones de la audiencia hostigada por la publicidad, pero resaltando además que el ciudadano es un espectador pasivo de la comunicación política (Canel, 2007). Incluso algunos medios de renombre internacional han titulado el proceso de gestión como "El milagro ecuatoriano"4.

Así lo certifica Esperanza Morales, autor de El discurso político de Rafael Correa, para quien "La imagen que Correa quiere construir en lo que dice y en lo que muestra (es decir, también con signos de carácter semiótico) es la de un personaje político moderno, que actúa de manera ética y cercana a los ciudadanos de las clases populares" (Morales, 2012). Una de las evidencias de esta cercanía fue la decisión de mantener y duplicar el bono de desarrollo humano o bono solidario, implementado en el gobierno de Jamil Mahaud (1998).

Correa Delgado además maneja un discurso que denota cercanía con el ciudadano común, al que llama: "compañerito", además del uso continuo de frases como: "la patria ya es de todos"; ¡Que no nos roben la esperanza!; ¡El fin de larga noche neoliberal!, marcan los elementos de convencimiento y persuasión que usa el Presidente de manera reiterativa. Hablamos de discurso funcional, que va más allá de la acción y de la interacción (Van Dijk, 1999) construyendo una clara relación de poder.

El papel de los medios de comunicación es fundamental para el mantenimiento de la democracia, pero a su vez, los medios tienen una relación de dependencia con

\footnotetext{
${ }^{4}$ La frase: " milagro ecuatoriano" es atribuida a William Black, catedrático y economista de la universidad de Missouri. Su intervención, fue réplica en algunos medios de comunicación. Disponible en:

http://www.elciudadano.gob.ec/intelectual-estadounidense-william-black-reconocio-el-milagro-ecuatoriano/ ( $12 / 04 / 2014)$.

REDMARKA UIMA-Universidad de A Coruña - CIECID

Año VIII, Número 14, (2015), v I pp. 75-101

http://www.redmarka.net/ ISSN 1852-2300
} 
el poder y logran transferir al público ciertos temas con el carácter de prioritario, bajo la lógica a la agenda-setting (McCombs; Maxwell, 1972) .

Los gobiernos, por su parte, no son actores pasivos. Todos los medios dependen, en mayor o menor medida, de recursos de publicidad oficial y de organismos para estatales (Sorj, 2000). Y los periodistas por su parte también tienen sus propias limitaciones, ante la respuesta a la disyuntiva sobre para "quién escriben los periodistas, si para los ciudadanos o para los políticos, del mismo modo que estos últimos hablan condicionados por los anteriores" (Ruás, 2011). Todo ello, teniendo en cuenta que la clásica división de poderes de Montesquieu, entre Legislativo, Ejecutivo y Judicial, ha provocado corrientes de autores críticos que, más allá de la consideración de la prensa y los medios en general como el "cuarto poder" o un "contrapoder" de los poderes establecidos, mencionan la existencia de dos únicos poderes, el económico y el mediático (Ramonet,1986), incluso con el segundo dependiendo del primero. Es importante resaltar la cercanía del periodista I. Ramonet con el régimen Correista, lo que ha provocado su visita a Ecuador en más de dos ocasiones.

Precisamente, en esta línea, este artículo buscar reflejar la influencia de los medios en la opinión pública; analiza de manera coyuntural el nacimiento de los medios públicos y la oposición de los medios de comunicación privados hacia el Gobierno, como elementos fundamentales en el diseño de los política comunicacional de Rafael Correa y el despliegue mediático de la misma.

En líneas generales, la cultura periodística en Ecuador, que estuvo marcada por la presencia única y monopólica de los medios privados, puede resumirse en tres puntos fundamentales:

1.- Un modo de hacer y producir (el régimen de propiedad y control)

2.- Un modo de pensar y actuar (las condiciones y exigencias bajo las cuales trabajan los periodistas)

REDMARKA UIMA-Universidad de A Coruña - CIECID Año VIII, Número 14, (2015), v I pp. 75-101 http://www.redmarka.net/ ISSN 1852-2300 
3.- Un modo de decir y narrar (los discursos y las prioridades informativas) (Abad, 2011)

A partir del 2007, la estructura mediática del país sufre algunos cambios que modifican los esquemas de producción informativa y de administración, soportando en algunos casos una crisis de representatividad y pérdida de legitimidad (Cerbino; Ramos, 2013). La comunicación en el sector público está constituyendo un negocio creciente en muchos países (Howlett, 2009, p. 23) y Ecuador no es la excepción.

La estrategia de comunicación del gobierno de Correa es una de las más consolidadas de América Latina, y el diálogo con el pueblo es una de sus fortalezas. Se caracteriza por un discurso que se difunden de manera privilegiada en el sistema de medios públicos y de acuerdo con la revista española El Molinillo No. 55 (Redoli, 2013, p. 15) vinculada con la Asociación de Comunicación Política (ACOP), nunca en Ecuador hubo una presencia comunicativa estatal como la que se observa en este proceso de la "revolución ciudadana".

El discurso activo de Correa es expresado por medio de "Enlaces Ciudadanos" que son retransmitidos desde el 20 de enero de 2007, a través de distintos soportes. Si bien su plataforma matriz es la televisión, se replica en buena parte de las radio estaciones del todo el país. En este espacio semanal de comunicación se tratan temas coyunturales y se rinde cuentas de las acciones gubernamentales a la ciudadanía, proceso que convierten a Correa en un "Estado Comunicador" (Rincón, 2010).

La presencia activa de Correa en los medios de comunicación, bajo la figura de rendición de cuentas, sobrepasa incluso la autoridad electoral representada en el país por el Consejo Nacional Electoral (CNE), organismo que decretó la veda electoral, sin embargo a ello, se realizó el Enlace 362, desde la ciudad de Quito. La comunicación gubernamental de Ecuador destaca por el uso eficiente de códigos mediáticos impactantes, por su lenguaje pedagógico y por una clara intención de hablar a las mayorías (Ramos, 2012), aproximándose a su 
cotidianidad y a sus prácticas. Estrategia que está reforzada por el uso constante y vinculante de slogans como: "La revolución ciudadana está en marcha" o "La patria ya es de todos". El gobierno basa su exitosa estrategia de comunicación política en la difusión de los enlaces sabatinos, las cadenas nacionales y la publicidad oficial, espacios que manejan una lógica de contacto directo con los ciudadanos, es una forma en la cual el Presidente se somete al escrutinio público5 . Las intervenciones del Presidente hacen que se perciba como el centro de la nación ( De la Torre, 2010).

Tanto las cadenas sabatinas denominadas "Enlaces Ciudadanos", como la publicidad y diálogos en vivo por televisión nacional, permiten mostrar las obras y acciones del gobierno de Correa, así como también el disgusto y rechazo directo con algunos medios de comunicación y periodistas que se han expresado como contrarios la política gubernamental.

Proceso que a su vez es replicado por los medios como una estrategia de defensa de su credibilidad. Medios de comunicación están siendo atacados no por su poder, sino a causa de su creciente fragilidad (Sorj, 2000).

Los enlaces son preparados desde la Secretaria Nacional de Comunicación6, organismo que ha diseñado un "Manual de Procesos Comunicacionales", que pretende que la información difundida esa concisa y específica.

\section{Metodología}

Para determinar el cambio del mapa mediático en Ecuador desde el año 2007, se hizo un mapeo cronológico/descriptivo, de los dos periodos presidenciales de Rafael Correa, resaltando los cambios trascendentales en la comunicación del país.

\footnotetext{
${ }^{5}$ Declaraciones textuales de Fernando Alvarado Secretario General de Comunicación, según entrevista realizada con fecha 28 de enero de 2009. En: Cerbino Mauro; Ramos Isabel. La comunicación de masas en tiempos de revolución ciudadana 2012, Recuperado el 24 de Octubre del 2013 de www.gumilla.org/biblioteca/bases/biblo/texto/COM2009145_34-39.pdf

${ }^{6}$ La Secretaria Nacional de Comunicación, creada en este Gobierno, tiene su página oficial en el siguiente enlace: www.secom.gob.ec. Recuperado el 18 de junio del 2013.
}

REDMARKA UIMA-Universidad de A Coruña - CIECID

Año VIII, Número 14, (2015), v I pp. 75-101

http://www.redmarka.net/ ISSN 1852-2300 
Este mapeo consistió en detallar, de manera concisa y cronológica, la creación de medios de comunicación públicos en Ecuador y su influye en la forma de comunicar a la sociedad. No olvidemos que gran parte de los medios públicos se consolidan como herencia de la crisis bancaria

Se tomó un antecedente previo realizado por Ciespal (2009) que sostiene que apenas el $37 \%$ de la población encuestada puede reconocer cuáles son los medios públicos en el país. La encuesta se hizo únicamente en las ciudades de Quito y Guayaquil (Ayala; Calvache, 2009).

Los datos analizados para el presente artículo provienen de una recolección de notas periodísticas de varios medios de comunicación, separando los hechos por orden de importancia y fechas. Si bien este trabajo parte de fuentes periodísticas, en su mayoría, son artículos publicados en las páginas Web de diarios de referencia en el país: El Universo, El Comercio, El Telégrafo, entre otras. También hace una revisión bibliográfica de la literatura disponible sobre la cuestión de carácter nacional e internacional en relación a la actividad política de Ecuador, artículos que han abierto un amplio debate sobre el quehacer de los medios y la responsabilidad de los periodistas en particular .

El objetivo de este artículo es determinar la evolución del discurso en los medios de comunicación, por parte del presidente Rafael Correa, por medios de las siguientes herramientas enlaces ciudadanos, apariciones en prensa, inversión publicitaria y accionar de los medios públicos.

En esta investigación, se analizaron también algunas alusiones del Presidente en cuanto a los medios de comunicación ecuatorianos denominados como independientes ( privados). Identificando varios altercados entre el poder y algunos periodistas, que han sido narrados por la prensa ecuatoriana, en todos sus niveles.

Los cambios que se han dado en el país, en materia de comunicación y política, permiten realizar un estudio detallado del gobierno de Correa y su influencia en la comunicación del continente.

REDMARKA UIMA-Universidad de A Coruña - CIECID Año VIII, Número 14, (2015), v I pp. 75-101 http://www.redmarka.net/ ISSN 1852-2300 


\section{Correa gana las elecciones:}

\section{Cambios en el mapa político y mediático del país}

En la campaña electoral del 2006 participaron representantes de 13 partidos políticos a la presidencia de Ecuador. Rafael Correa Delgado, representante del movimiento Alianza País - Patria Altiva y Soberana PAís (movimiento que sirve como plataforma política a la Revolución Ciudadana impulsada por Correa) llegó a la segunda vuelta con Álvaro Noboa, candidato por el Partido Renovador Institucional de Acción Nacional (PRIAN).

El día 26 de noviembre del 2006, el Tribunal Supremo Electoral confirmó el triunfo del binomio Correa/Moreno, con el $56,4 \%$ de votos, sobre el candidato por el partido PRIAN, Álvaro Noboa, quien obtuvo el $43,6 \%$.

Si bien es cierto el partido político del candidato elegido es un proyecto nuevo, su estabilidad depende del mismo presidente, quien ha recibido un apoyo en la urnas destacado. Este proyecto político busca: "una sociedad más incluyente, responsable en términos políticos y activa en la gestión del poderes" ( Jaramillo; Jassir, 2013) vinculando sectores tradicionalmente olvidados como los discapacitados por medio del programa: "Misión Manuela Espejo", y a las mujeres, mediante el ascenso a órganos de burocracia y gestión.

El perfil político de Correa llamó la atención de los medios el año 2005, cuando desempeñaba sus funciones como ministro de Economía en el gobierno de Alfredo Palacio (2005). Desde aquel tiempo ya se evidenció un acercamiento con Venezuela: Otra de las acciones que perfilaron una marcada posición de lo que

REDMARKA UIMA-Universidad de A Coruña - CIECID Año VIII, Número 14, (2015), v I pp. 75-101 http://www.redmarka.net/ ISSN 1852-2300 
se llamó la nueva izquierda, que ha sido promocionada como el Socialismo del Siglo XXI7.

Desde que Correa está en el poder, el acercamiento mediático con Venezuela ha sido evidente. TeleSUR "La señal informativa de América Latina" es un canal interamericano impulsado por el gobierno venezolano que, en conjunto con otros países Sudamericanos, busca "liderar y promover los procesos de unión de los pueblos del SUR". Entendiéndose el sur como aquellos países que comparten "la lucha de los pueblos por la paz, autodeterminación, respeto por los Derechos Humanos y la Justicia Social", según se lee en la Misión de este medio en su página de Internet 8.

Según el sitio EcuRed, TeleSUR9 surgió en enero del 2005, y fue aprobado por el Consejo de Ministros de Venezuela, que aportó un 70\% de capital. El 30\% restante fue aportado por Argentina, Bolivia, Cuba, Ecuador y Nicaragua.

En el caso Ecuador y de acuerdo con información del diario El Comercio publicada el 2 de noviembre del 201110, TeleSUR comenzó sus transmisiones en televisión en abierto en el 2011 (antes sólo se podía hacer sólo por cable). Desde octubre del 2007, indica que Ecuador funge como accionista del $5 \%$.

En el 2000 se realizó una Consulta Popular, que obtuvo la aprobación para la creación de una Asamblea Constituyente, con la misión de redactar nueva Constitución. El mandato de la Constitución estableció que la Asamblea Nacional aprobase un cuerpo legal para una Ley de Comunicación, y en septiembre del 2009 se creó una Comisión Especial Ocasional de Comunicación para analizar tres proyectos relacionados con de Ley Orgánica de Comunicación:

\footnotetext{
${ }^{7}$ La propuesta del " socialismo del siglo XXI" surgió en la Venezuela Bolivariana que ha influido en el socialismo cubano y en los países del Alba de América Latina

${ }^{8}$ El canal de televisión venezolano mantiene activa la siguiente página web: www.telesurtv.net

${ }^{9}$ ECUARED "Telesur" (s.f). Recuperado el 13 de enero del 2013. http://www.ecured.cu/index.php/TeleSUR

10 "En Ecuador la señal de Telesur ya es abierta", Diario El Comercio, (s.f). Recuperado el 28 de febrero del 2013 de http://www.elcomercio.com/politica/Ecuador-senal-Telesur-abierta_0_583141893.html

REDMARKA UIMA-Universidad de A Coruña - CIECID

Año VIII, Número 14, (2015), v I pp. 75-101

http://www.redmarka.net/ ISSN 1852-2300
} 
El proyecto de ley se discutió en el país desde el 2009, y fue aprobado el junio del 2013, por una mayoría casi absoluta, más 100 votos en relación a los 137 asambleístas votantes. El Reglamento General a la Ley Orgánica de Comunicación a través del Decreto No. 214, en enero del 2014.

Estos articulados de orden jurídico a su vez dieron paso a la consolidación de la Superintendencia de la información y la Comunicación, cuyas atribuciones como un organismo técnico, se pueden resumir así: "vigilancia, auditoría, intervención y control, con capacidad sancionatoria, de administración desconcentrada, con personalidad jurídica, patrimonio propio y autonomía administrativa, presupuestaria y organizativa; que cuenta con amplias atribuciones para hacer cumplir la normativa de regulación de la Información y Comunicación"11.

El organismo desde su creación a principios del 2014 está en manos del periodista Carlos Ochoa, periodista de larga trayectoria en la televisión ecuatoriana y muy cercano al grupo de gobierno.

Desde el inicio de su primer mandato, el gobierno de Correa tuvo varios enfrentamientos con medios de comunicación. Por ejemplo el caso de la suspensión por tres días de la estación de televisión Teleamazonas, en el 2009, por la transmisión de una noticia basada en supuestos. También se dio por terminada la concesión por parte de la Superintendencia de Telecomunicaciones, de Radio Arutam (frecuencia 107.3 MHz, desde la ciudad de Sucúa, en el interior de la selva ecuatoriana) por incitación a la violencia durante una manifestación popular en la ciudad de Macas, provincia de Morona Santiago.

Los enfrentamientos de Correa con la prensa han involucrando a su familia. Fabricio Correa, hermano mayor del presidente de Ecuador, fue denunciado en 2009 por los periodistas Juan Carlos Calderón y Christian Zurita,

\footnotetext{
${ }^{11}$ Referencia textual tomada de la página web del Consejo de Participación Ciudadana y Control Social. Disponible en: http://www.cpccs.gob.ec ( 22/05/2014)

REDMARKA UIMA-Universidad de A Coruña - CIECID

Año VIII, Número 14, (2015), v I pp. 75-101

http://www.redmarka.net/ ISSN 1852-2300
} 
debido a que contenía contratos de millones de dólares con el Estado 12, resumidos en el libro titulado: "El gran hermano".

Rafael Correa los demandó en 2011 por daños morales. Los periodistas decían que más allá de evidenciar que el Presidente sabía de estos movimientos, querían demostrar cómo funcionaba el gobierno ecuatoriano. Para ellos, el proceso legal iniciado por del Presidente era, claramente, una cortina de humo para deslindarse de las acciones del hermano, al que no denunció por "respeto" a su madre. Correa los demandó por 10 millones de dólares americanos y el expediente fue paralizado.

Correa también tuvo enfrentamientos legales con el diario La Hora, medio al cual demandó en el 2007, por la publicación de un editorial titulado "Vandalismo Oficial". La base de la denuncia está en Art. 230 del Código Penal de Ecuador, que impone multas y hasta dos años de prisión para el causante que exprese "amenazas o injurias que ofendan al presidente". Este no ha sido el único enfrentamiento con el grupo empresarial/familiar Vivanco.

El juicio que el presidente Correa presentó contra el periodista Emilio Palacio, en 2011, a los directores del diario ecuatoriano El Universo, y contra la compañía anónima El Universo, que edita Diario El Universo, quizás fue uno de los más polémicos de su administración. Por un artículo de opinión firmado por Emilio Palacio, "No a las mentiras", publicado El Universo el 6 de febrero de 2011, los acusa de delito de injuria grave a la autoridad. En su querella el presidente pidió al Juez el pago de 80 millones de dólares americanos como indemnización civil. El proceso de conflicto permanente entre gobierno y medios se suceden, de modo inédito en la historia de la democracia ecuatoriana, juicios y sanciones a medios, entre ellos al canal de televisión Teleamazonas, y los diarios El

\footnotetext{
${ }^{12}$ cfr. "Rafael Correa demanda por US\$10 millones a dos periodistas ecuatorianos", en América Economía, 2011, Recuperado el 23 de mayo de 2013 de http://www.americaeconomia.com/politicasociedad/politica/rafael-correa-demanda-por-us10-millones-dos-periodistas-ecuatorianos
}

REDMARKA UIMA-Universidad de A Coruña - CIECID

Año VIII, Número 14, (2015), v I pp. 75-101

http://www.redmarka.net/ ISSN 1852-2300 
Universo 13 y La Hora 14, por citar sólo algunos de los más relevantes, son características comunes de la disputa.

Por otro lado, los medios públicos, desde su creación aproximadamente en el 2008, han cumplido con el deber de informar de cada una de las obras del gobierno de Correa, haciendo de las fuentes oficiales la perspectiva más presente.

\section{Mecanismos de difusión del discurso Correísta}

Desde el 20 de enero del 2007, los ecuatorianos ven y escuchan a Rafael Correa en el "Enlace Ciudadano", programa sabatino que tiene una duración de tres horas aproximadamente. En este espacio semanal de comunicación, se tratan temas coyunturales y se rinde cuentas de las acciones gubernamentales a la ciudadanía.

A este enlace se han sumado otras estrategias de comunicación, como la intervención del presidente en los medios públicos, y activa presencia por medio de su cuenta oficial en Twitter @MashiRafael, entre otras. La cuenta a inicios del 2014, registró 1.5 millones de seguidores.

\subsection{Publicidad del Estado}

En junio del 2009, Rafael Correa firmó el decreto $\mathrm{N}^{\circ}$. 1793, que modifica la Ley de Contratación Pública. Con esto prohíbe al Estado la celebración de contratos con empresas que tengan domicilio en paraísos fiscales, causando un fuerte golpe a varios medios de comunicación como el diario La Hora, vinculado con paraísos fiscales en Bahamas y Gran Bretaña; diario El Universo en las Islas Gran Caimán; El Comercio, con 49\% de participación en las Islas Vírgenes-Estados Unidos y Panamá; y la revista Vanguardia, 55\% vinculado a empresa de Colombia

\footnotetext{
${ }^{13}$ Diario El Universo es uno de los diarios de referencia del país, se publica en la ciudad de Guayaquil desde 1921. Este órgano informativo se ha mostrado contrario al régimen.

${ }^{14}$ Diario La Hora, es un diario de nacional con ediciones regionales en casi todas las provincias del Ecuador. Se publica desde 1982, su posición critica con el régimen les ha causado más de un conflicto.
}

REDMARKA UIMA-Universidad de A Coruña - CIECID

Año VIII, Número 14, (2015), v I pp. 75-101

http://www.redmarka.net/ ISSN 1852-2300 
y de las Islas Vírgenes (Punín, 2011). Después Luego de la firma del Decreto, "EI Universo, El Comercio, La Hora, Expreso y la revista Vanguardia, han visto disminuida la cuota publicitaria por parte del Gobierno."

A causa de aquel Decreto, el grupo Vivanco, conformado por La HoraVanguardia, no recibe publicidad de organismos de gobierno. La medida adoptada provocó que varios medios de comunicación legalicen sus acciones y que las inversiones en el extranjero regresen al país. De hecho el primer medio en anunciar su cierre tras de la aprobación de la Ley de Comunicación fue revista de investigación dedicada a temas políticos y de gobierno denominada: Vanguardia.

Según Fundamedios15, los gastos en publicidad de la Presidencia en el 2009 fueron de \$, 4.919.584,45. La organización sin fines de lucro, aglutina a algunos periodistas vinculados con los medios de comunicación social recibe de manera continua el rechazo del presidente.

A la vez que disminuyó el aporte publicitario del gobierno a los medios contrarios, otros medios afines vieron aumentar su cuenta en publicidad. Radio La Luna, medio de comunicación que impulsó la conformación de "Los forajidos 16", y liderada por el radiodifusor Paco Velasco, aumentó considerablemente el rubro en publicidad, "entre el 2007 y el 2009.

La Luna recibió contratos de entidades públicas por más de USD. 876 mil. En el 2007 Velasco fue electo asambleísta del partido de Gobierno, Alianza País (AP), actualmente es Ministro de Cultura, cargo de designación expresa por parte del Presidente.

Desde el 2009, los ingresos económicos de Velasco y La Luna aumentaron sustancialmente. Mientras en el 2005 Velasco pagó USD 75 de Impuesto a la

\footnotetext{
${ }^{15}$ Fundamedios es una organización de la sociedad civil que apoya el desarrollo de medios de comunicación democráticos y el ejercicio del periodismo de calidad, formado por periodistas y comunicadores del Ecuador. Su página web oficial es: http://www.fundamedios.org/. Es además uno de los organismos que mayores criticas ha recibido del régimen por sus mecanismos de financiamiento.

${ }^{16}$ Los forajidos, es una agrupación ciudadana que participó en la caída del presidente Lucio Gutiérrez en abril del 2006, que se originó en la ciudad de Quito, tras la convocatoria del radiodifusor Paco Velasco.
}

REDMARKA UIMA-Universidad de A Coruña - CIECID

Año VIII, Número 14, (2015), v I pp. 75-101

http://www.redmarka.net/ ISSN 1852-2300 
Renta (IR), en el 2006 pagó USD 1 671. La Luna, que el 2005 declaró USD 533, el siguiente año pagó USD 3.213. A principios del 2011 la radio cambió de nombre y línea informativa, para dedicarse al deporte y al entretenimiento, en particular". 17 La Unidad de Investigación del Diario El Comercio señala que Velasco dejó de ser parte de la Radio la Luna, en el 2009, justamente por su participación política en la Asamblea.

En el 2008, según informe de Infomedia/lbope, citado libro denominado: Palabra Rota (Ricaute, 2010) la publicidad oficial supuso 14.894 .000 de dólares americanos, y aumentó en los dos años siguientes: en el 2009 alcanzó los 40 millones de dólares y en 2010 los 60 millones, teniendo a la televisión como el principal soporte para difusión de mensajes gubernamentales.

\section{Medios públicos, medios en Ecuador}

\subsection{Estatalización del diario El Telégrafo}

Entendemos como medios públicos al sistema mediático paralelo al sistema de medios comerciales. Los públicos ofrecen una serie de herramientas para satisfacer el derecho a la información del público, en general (Navarro; Villanueva, 2010) y por que su propia misión y naturaleza son distintos a los medios estatales. Los medios públicos se caracterizan por ser universales, diversos e independientes (Unesco, 2001).

En el caso particular de Ecuador, la historia de los medios públicos inicia con la estatización de diario El Telégrafo fue un proceso largo y complicado. El diario perteneció hasta el 2002 al ex banquero Fernando Aspiazu, mayor accionista y presidente del Banco del Progreso. Cuando este banco quebró, generó pérdidas al Estado y a miles de depositantes. En ese mismo año, las acciones de El Telégrafo fueron incautadas por la Agencia de Garantía de Depósitos

\footnotetext{
${ }^{17}$ La Luna recibió contratos oficiales. Diario El Comercio (s.f). Recuperado el 28 de Octubre del 2013 de http://www.elcomercio.com/politica/Luna-recibio-contratos-oficiales-USD_0_406759376.html

REDMARKA UIMA-Universidad de A Coruña - CIECID

Año VIII, Número 14, (2015), v I pp. 75-101

http://www.redmarka.net/ ISSN 1852-2300
} 
(AGD), institución que tomó posesión del 99,6\% de las acciones de El Telégrafo (mientras uno de los accionistas, Carlos Navarrete, se quedó con el 0,3\%) para devolver acreencias a los perjudicados.

En los años 2002 y 2004 hubo intentos fallidos de subastar el diario, y mientras la AGD diseñaba un plan para subastarlo y Navarrete asumió la dirección del medio. En el año 2005, Navarrete Castillo18 se había convertido en el principal accionista del medio luego de un aumento de capital que, posteriormente, fue declarado ilegítimo. Así, en junio del 2007, avalado por una resolución de la Superintendencia de Compañías, el Estado ecuatoriano, con el Ministerio de Finanzas como principal accionista, retomó el control del diario con nueva imagen y bajo nueva dirección editorial. El 17 de marzo de 2008 El Telégrafo aparece con el eslogan de "Primer diario público del Ecuador".

Diario Hoy informó que en el 2009 se imprimían treinta mil ejemplares de El Telégrafo, pero veintiséis mil de las cuales se obsequiaba en instituciones públicas y privadas. El objetivo principal era que a fines de ese año se distribuyan 39 mil periódicos diarios. Se concretó la compra de una nueva rotativa en alrededor de \$6,5 millones a la empresa alemana Koening \& Bauer AG (KBA), que empezaría a trabajar en diciembre. Así, el gobierno de Rafael Correa destinó ese año a El Telégrafo un presupuesto de $\$ 9$ millones, que se suman a los $\$ 7$ millones invertidos en 2008, año en que comenzó la administración estatal.

Según una publicación del 2011 del diario El Universo, \$ 75 mil en multas tuvo que cancelar diario El Telégrafo por retrasos en los pagos a la empresa alemana Koening \& Bauer AG (KBA), proveedora de su nueva rotativa19. Vinicio Alvarado, secretario de la Administración, en su calidad de coordinador designado de la

\footnotetext{
${ }^{18}$ Carlos Navarrete Castillo, fue el director de Diario El Telégrafo antes de pasar a ser medio público.

${ }^{19}$ Presupuestos de El Telégrafo del 2007 y 2008 los aprobó Alvarado. (s.f) Recuperado el 23 de febrero de 2013 alvarado.html

REDMARKA UIMA-Universidad de A Coruña - CIECID Año VIII, Número 14, (2015), v I pp. 75-101 http://www.redmarka.net/ ISSN 1852-2300
} http://www.eluniverso.com/2011/05/31/1/1355/presupuestos-telegrafo-2007-2008-aprobo- 
Presidencia, aprobó cada presupuesto anual del diario durante los ejercicios del 2007 y 2008, siendo el funcionario gubernamental que apoyaba la gestión ante el Ministerio de Finanzas. En tres años, El Telégrafo perdió \$13 millones20.

\subsection{Los primeros medios públicos}

Radio Pública antes denominada Radio Nacional retoma su señal a mediados de noviembre del 2007. La Radio Pública de Ecuador (RPE) es un medio de comunicación ecuatoriano creado en los años 40 del siglo pasado con el pretendido objetivo de "precautelar intereses y visiones generales de carácter nacional, más que intereses y visiones particulares" según el artículo "Percepción sobre los medios públicos en Ecuador" del CIESPAL de 2009 ( Ayala; Calvache, 2009).

Los medios públicos buscan "brindar a la ciudadanía contenidos que les formen, informen y entretengan" de manera sana así como fomentar y fortalecer los valores "familiares, sociales, culturales y la participación ciudadana", según la Misión de esta empresa. RPE, al igual que Ecuador TV (ECTV), fue creada el 29 de diciembre de 2009 vía Decreto Presidencial № 196.

En Caracas se suscribe un contrato del préstamo no reembolsable por 5 millones de dólares entre el Gobierno de Ecuador y el Banco de Desarrollo Económico y Social (Bandes) de Venezuela, para financiar el proyecto de Televisión Pública de Ecuador, y así Ecuador TV se estableció en octubre del 2007. El canal fue establecido como cadena publica, que trasmite contenidos de productoras independientes locales e internacionales, tales como documentales e informativos televisivos de Discovery Channel, Discovery Kids, TVE, la BBC, DW, TV5 Monde,

\footnotetext{
${ }^{20}$ Aquí se puede leer la versión pública del medio oficial en relación al proceso empresarial interno. "El Telégrafo a la ciudadanía", Diario El Telégrafo (s.f) recuperado el 13 de enero del 2013 (www.partealta.ec) de http://www.partealta.ec/noticias/economia-solidaria/7164-el-telegrafo-a-la-ciudadania
}

REDMARKA UIMA-Universidad de A Coruña - CIECID Año VIII, Número 14, (2015), v I pp. 75-101 http://www.redmarka.net/ ISSN 1852-2300 
RAI, Voz de América, ViVe y TeleSUR. Utiliza el eslogan "A partir de ahora la televisión ya es de todos".

Tanto Ecuador TV como Radio Pública son operados por la Empresa Pública Radio y Televisión de Ecuador (RTVECUADOR S.A.), y su objetivo es la producción, programación y transmisión de contenidos televisivos y radiofónicos. Para ello, cuenta con oficinas en Quito, Cuenca, Puyo y Guayaquil.

A finales del 2009, se creó, a través de un decreto ejecutivo suscrito, una nueva entidad pública cuyo patrimonio está conformado por los bienes muebles de la sociedad anónima Televisión y Radio del Ecuador S.A. (RTV Ecuador), que comprende Radio Pública del Ecuador (RPE) y Ecuador TV. Para la creación de la nueva Empresa Pública Televisión y Radio de Ecuador (E.P. RTVEcuador), se declaró la disolución forzosa de RTV Ecuador S.A., y se dispuso la transferencia de su patrimonio a la recién nacida empresa pública. Su patrimonio, conformado por los bienes muebles de la sociedad anónima RTV Ecuador S.A., asciende a\$. 3'307.893,96.

\subsection{Incautación de medios del Grupo Isaías}

La AGD incautó 195 empresas del grupo económico para que el Estado pudiera recuperar 661 millones de dólares de la deuda por las quiebra del banco Filanbanco que se produjo en 1999. Entre las empresas incautadas están los canales de televisión Gamavisión, Cablevisión y TC Televisión, acciones de un sistema de cable, TV Cable, dos revistas, La Onda (juvenil) y El Agro y dos radioemisoras, Carrusel y Super $\mathrm{K}$.

Al momento de la incautación, los portavoces vinculados al Gobierno afirmaron que los medios incautados serían vendidos, pero hasta la fecha no se ha consolidado el proceso.

REDMARKA UIMA-Universidad de A Coruña - CIECID

Año VIII, Número 14, (2015), v I pp. 75-101

http://www.redmarka.net/ ISSN 1852-2300 
El Ciudadano (2008) es el medio de comunicación oficial de la Presidencia de la República del Ecuador. Cuenta con una edición digital y otra impresa. Bajo el slogan de "El Periódico del Gobierno de la Revolución Ciudadana", difunde entrevistas, reportajes y noticias de actualidad respecto de la labor que realiza el Gobierno Nacional. La versión impresa se distribuye de manera gratuita en todo el país, a través de Correos del Ecuador y de las entidades públicas, no se han hecho público los niveles de lectoría, ni de impacto del mencionado producto.

El Ciudadano se desprende el programa dominical El Ciudadano TV que es transmitido por Ecuador TV y retransmitido por Cablevisión, y El Ciudadano Radio, que es un espacio noticioso que forma parte del sistema informativo de la Secretaría Nacional de Comunicación, el cual es transmitido de lunes a viernes por Radio Universal.

En el 2009, nació la Agencia de Noticias de Ecuador y Suramérica (ANDES), financiada con recursos del Estado. ANDES es una empresa informativa pública que dice "dar espacio a todos los actores políticos, sociales y económicos, sobre todo a aquellos que no tienen espacio en los otros medios de comunicación". Nació bajo el impulso de la Secretaría Nacional de Comunicación y actualmente depende de Ecuador TV y los otros medios creados con iguales fines. Su circulación es únicamente en la Red.

\section{Conclusiones}

REDMARKA UIMA-Universidad de A Coruña - CIECID

Año VIII, Número 14, (2015), v I pp. 75-101

http://www.redmarka.net/ ISSN 1852-2300 
Una jugada estratégica en este gobierno han sido los "Enlaces Sabatinos", en la cual los ciudadanos escuchan todos los sábados la rendición de cuentas públicas por parte del presidente y ocasionalmente de su equipo de gobierno, estrategia que muestra la capacidad de persuasión del presidente ecuatoriano, genera un proceso de cercanía constante, recurso que no fue usado autoridades anteriores en similares cargos. A ello se suma el índice de popularidad del presidente, que han sido ratificados en el apoyo popular en las urnas.

El significado ideológico que construye Correa en cada una de sus intervenciones influye directamente en la opinión pública ecuatoriana. Este es el escenario mediático aprovechado por el presidente Correa para hablar continuamente sobre la libertad de expresión, manipulación mediática, y otras acciones que están relacionadas con los grupos tradicionales de poder en Ecuador.

Además, el poder casi absoluto que tenían los medios de comunicación privados en Ecuador, ha sido debilitado, tal y como se puede observar en este artículo, como consecuencia del proceso descrito. La creación y refuerzo de los medios públicos ha permitido una difusión masiva del discurso de Correa y de los otros estamentos de gobierno.

A partir de la incautación de las empresas del grupo Isaías, y el juicio contra el diario El Universo y los periodistas Emilio Palacio, Juan Carlos Calderón y Cristiam Zurita y la aprobación del Ley Orgánica de Comunicación, la política de comunicación en el país ha cambiado, al igual que la posición de los medios frente al quehacer ejecutivo.

Otro elemento de fondo, es la capacidad de inversión en publicidad que tiene el gobierno, que actúa con un elemento de control hacia los medios de comunicación. Distribución que podría cambiar si se aplica el Art. 95 y 96 de la comentada Ley.

REDMARKA UIMA-Universidad de A Coruña - CIECID

Año VIII, Número 14, (2015), v I pp. 75-101

http://www.redmarka.net/ ISSN 1852-2300 


\section{Bibliografía:}

Abad, G. (2011). El Club de la pelea. Gobierno y medios, un entramado de fuerzas y debilidades. Quito, Veeduría Ciespal, № 5 .

Ayala, A ; Calvache, M. (2009). "Percepción sobre los medios públicos en Ecuador", Informes de investigación. Quito, Ciespal. [Recuperado 10-8-2014] de http://cracketzaa-tv.blogspot.com/

Canel, M. J. (2007). La comunicación de las instituciones públicas. Madrid, Tecnos

Cerbino, M ; Ramos, I. (2009). Apuntes para la democratización del espacio mediático en Ecuador, Comunicación, Espectro América, Asociación de Periodistas Europeos (APE) / Fundación de Nuevo Periodismo Iberoamericano Lisboa, Portugal. [Recuperado 02-05-2014] de http://www.gumilla.org/biblioteca/bases/biblo/texto/COM2009145_34-39.pdf Cerbino, M ; Ramos, I. (2012). La comunicación de masas en tiempos de revolución ciudadana. Gumilla. [Recuperado 07-10- 2014] de www.gumilla.org/biblioteca/bases/biblo/texto/COM2009145_34-39.pdf

Consejo Nacional Electoral. Resultados elecciones seleccionales 2013 http://resultados2013.cne.gob.ec/Results.html?RacelD=1\&UnitID=1\&IsPS=0\&Lan $g \mid D=0$

Consejo de Participación Ciudadana y Control Social. [Recuperado 12-10- 2014] de http://www.cpccs.gob.ec

Corporación Latibarómetro. Informe. 2011. Santiago de Chile, p. 107 -112. [ Recuperado 06-11- 2014] de http://alertacontraelracismo.pe/wpcontent/uploads/2013/01/INFORME-DE-LATINOBAROMETRO-2011.pdf

REDMARKA UIMA-Universidad de A Coruña - CIECID Año VIII, Número 14, (2015), v I pp. 75-101 http://www.redmarka.net/ ISSN 1852-2300 
De la Torre, C. (2010). Rafael Correa un Populista del Siglo XXI, 2010.

[Recuperado 09-10-2014] de

http://lanic.utexas.edu/project/etext/lilas/vrp/delatorre.pdf

"Ecuared", Telesur (s.f) 2013. [Recuperado 10-11-2014] de http://www.ecured.cu/index.php/TeleSUR

"En Ecuador la señal de Telesur ya es abierta", El Comercio (s.f). (2013). [Recuperado 07-17- 2014] de http://www.elcomercio.com/politica/Ecuador-senalTelesur-abierta_0_583141893.html

"El Telégrafo a la ciudadanía”, El Telégrafo (s.f). [Recuperado 11-10- 2014] de http://www.partealta.ec/noticias/economia-solidaria/7164-el-telegrafo-a-laciudadania

Freidenberg, F.(2013). El Flautista de Hammelin: liderazgo y populismo en la democracia ecuatoriana. El retorno del pueblo. Populismo y nuevas democracias en América Latina, Editorial Carlos de la Torre y Enrique Peruzzotti, Quito, FLACSO, 2008 185-233 [Recuperado 11-12- 2014] de http://works.bepress.com/flavia freidenberg/21

Howlett, M. (2009). Government communication as a policy tool: A framework for analysis, The Canadian Science Review, 3(2) p. 23

Jaramillo, M y Jassir A. (2008). La revolución democrática de Rafael Correa, Centro de Estudios Políticos e Internacionales - CEPI-. Facultades de Ciencia Política y Gobierno y de Relaciones Internacionales, Bogotá, Editorial Universidad del Rosario. [Recuperado 11-14- 2014] de

REDMARKA UIMA-Universidad de A Coruña - CIECID Año VIII, Número 14, (2015), v I pp. 75-101 http://www.redmarka.net/ ISSN 1852-2300 
http://www.urosario.edu.co/urosario_files/f7/f74e53f8-7c2b-43bf-894cc4667318aa56.pdf.

"La Luna recibió contratos oficiales". Diario El Comercio (s.f). [Recuperado 11-162014] de http://www.elcomercio.com/politica/Luna-recibio-contratos-oficialesUSD_0_406759376.html

"Los bienes de la naciente empresa valorados en más de $\$ 3,3$ millones", Diario El Universo (s.f). [Recuperado 02-12- 2014]de http://www.eluniverso.com/2010/01/08/1/1355/bienes-naciente-empresavalorados-mas-millones. $h$ tml $p=1355 A \& m=1548$ "Ministerio de Telecomunicaciones presidirá radio y televisión públicas", Diario El Universo (s.f) [Recuperado 11-15- 2014] de http://www.eluniverso.com/2010/01/08/1/1355/ministerio-telecomunicacionespresidira-radio-television-publicas.html

Manual de calidad: Televisión y Radio de Ecuador. [Recuperado 08-13- 2014] http://www.rtvecuador.ec/imagesFTP/7770.MAC_RTVECUADOR.pdf.

MCcombs, M y Evatt D. (1972). The agenda setting Function of Mass Media en Public Opinion Quarterly.

Navarro, F; Villanueva E. (2010). Medios de servicio público y transparencia: análisis y medición de su desempeño. Intiyan Ciespal

Morales, E. (2012). El discurso político de Rafael Correa (Presidente de Ecuador), Revista de Estudios Filológicos, No. 23, Julio 2012 ISSN 1577-6921, [Recuperado 11-19-2014] de

REDMARKA UIMA-Universidad de A Coruña - CIECID Año VIII, Número 14, (2015), v I pp. 75-101 http://www.redmarka.net/ ISSN 1852-2300 
http://www.um.es/tonosdigital/znum23/secciones/monotonosmorales_lopez_correa_para_tonos_digital_2012.htm

Observatorio de Radiodifusión Pública en América Latina 2012. [Recuperado 1018-2014] de http://es.observatorioradiodifusao.net.br/index.php?option=com_content\&view=arti cle\&id $=98 \&$ Itemid $=142$.

"Presupuestos de El Telégrafo del 2007 y 2008 los aprobó Alvarado". Diario El Universo (s.f) [Recuperado 09-14- 2014] de http://www.eluniverso.com/2011/05/31/1/1355/presupuestos-telegrafo-2007-2008aprobo-alvarado.html.

Punín, M. (2011). Rafael Correa y la prensa ecuatoriana. Una relación de intrigas y odios. Razón y Palabra, núm. 75, febrero-abril 2011. [Recuperado 04-10- 2014] de http://www.redalyc.org/articulo.oa?id=199518706070.

Rafael Correa demanda por US $\$ 10$ millones a dos periodistas ecuatorianos. En Revista América Economía, 2011, [Recuperado 08-10- 2014] de http://www.americaeconomia.com/politica-sociedad/politica/rafael-correademanda-por-us10-millones-dos-periodistas-ecuatorianos.

Ramonet, I. (1986). La tiranía de la comunicación, Madrid, Debate.

Ramos, Isabel. 2012. La contienda política entre los medios privados y el gobierno de Rafael Correa. Utopía y Praxis Latinoamericano, No. 58 2012. [ Recuperado 07-12- 2014 ] de http://revistas.luz.edu.ve/index.php/upl/article/viewFile/10735/10387

REDMARKA UIMA-Universidad de A Coruña - CIECID Año VIII, Número 14, (2015), v I pp. 75-101 http://www.redmarka.net/ ISSN 1852-2300 
Redoli, David. 2013. La comunicación política debe ir de la mano del fortalecimiento de los partidos. El Molinillo, número 55, junio, pp. 13-26.

Ricaute, César. 2010. La palabra rota. Seis investigaciones sobre el periodismo ecuatoriano, Quito. Fundamedios.

Rincón, Omar. 2010. ¿Por qué nos odian tanto? Estado y medios de comunicación en América Latina, Centro de competencia en comunicación para América Latina.

Ecuador con acciones en Telesur. Diario El Universo (s.f)

Rt Ecuador, Televisión y Radio del Ecuador, (s.f) (2013) [Recuperado: 09-142014] de http://www.rtvecuador.ec/imagesFTP/7770.MAC_RTVECUADOR.pdf.

Ruás, José. 2011. Manual del candidato electoral, Catarata, Madrid

"Se archiva juicio de Rafael contra periodistas de El Gran Hermano", Diario EL Universo (s.f). [Recuperado: 06-11-2014] de http://www.eluniverso.com/2012/04/18/1/1355/archiva-juicio-presidente-rafaelcorrea-contra-periodistas-gran-hermano.html

"Secretaría Nacional de Comunicación", (s.f) Enlace ciudadano. [Recuperado 0811-2014] de

http://www.comunicacion.gob.ec/enlace-ciudadano/

Sorj, Bernardo. 2000, Poder Político y medios de comunicación. De la representación política al realty show. Poder político y medios de comunicación

Van Dijk. 1999, El análisis crítico del discurso, Barcelona, Anthropus.ERT

REDMARKA UIMA-Universidad de A Coruña - CIECID

Año VIII, Número 14, (2015), v I pp. 75-101

http://www.redmarka.net/ ISSN 1852-2300 
Unesco 2001, WRTVC, Publica Broadcasting: How? Why?, Paris

REDMARKA UIMA-Universidad de A Coruña - CIECID

Año VIII, Número 14, (2015), v I pp. 75-101

http://www.redmarka.net/ ISSN 1852-2300 